\title{
Review \\ Study designs for evaluation of combination treatment: focus on individual patient benefit
}

\author{
Martin C. Michel ${ }^{1}{ }^{*}$ and David Staskin ${ }^{2}$ \\ 1 Dept. of Pharmacology, Johannes Gutenberg University, Mainz, Germany; marmiche@uni-mainz.de \\ 2 Boston University School of Medicine, St. Elizabeth Medical Center, Boston, MA, USA; staskinatt@g- \\ mail.com \\ * Correspondence: marmiche@uni-mainz.de
}

\begin{abstract}
Combination treatment, i.e., the use of two or more drugs for the same condition, is frequent in medicine if monotherapy yields an insufficient therapeutic response. We here review and challenge clinical study designs and formats of reporting outcomes for the evaluation of the benefit/risk ratio of combination treatment over monotherapy. We demonstrate that benefits of combination treatment at the group level over-estimate the probability of benefit at the single patient level based on outcome simulations under almost any imaginable setting. Based on these findings we propose that studies testing combination treatment should always report on percentages of responders to monotherapy and combination treatment. We provide equations that allow calculation of the percentage of patients truly benefitting from combination (responders to both monotherapies) and that of patients exposed to risk of harm from adverse effects without a reasonable expectation of individual benefit. These considerations are explained based on real clinical data, mostly from the field of functional urology (male lower urinary tract symptoms).
\end{abstract}

Keywords: combination treatment; monotherapy; clinical trial design; benefit/risk assessment

\section{Introduction}

Combination treatment, i.e., the concomitant use of two or more drugs for the same indication, is frequently considered when administration of a single drug yields an insufficient therapeutic response. Guideline-recommended examples of this are the combination of a diuretic and a $\mathrm{Ca}^{2+}$ entry blocker in the treatment of arterial hypertension [1], of an inhibitor of the renin-angiotensin system, a $\beta$-adrenoceptor antagonist, a mineralocorticoid receptor antagonist and a sodium-glucose transporter 2 inhibitor in the treatment of heart failure with reduced ejection fraction [2], of a glucocorticoid and a $\beta_{2}$-adrenoceptor agonist in the treatment of asthma [3], of various types of agents in oncology [4], of a muscarinic receptor antagonist and a $\beta_{3}$-adrenoceptor agonist in the treatment of overactive bladder syndrome [5], or of an $\alpha_{1}$-adrenoceptor antagonist and a $5 \alpha$-reductase inhibitor in the treatment of male lower urinary tract symptoms (LUTS) attributed to benign prostatic hyperplasia [6]. This paper aims to review and challenge the rationale behind frequently used study designs and reporting formats in the evaluation of combination treatment as compared to monotherapy. We will illustrate the discussed concepts largely with examples from the field of functional urology (male LUTS) but propose that our considerations will be applicable to many if not most areas of medicine; a systematic review of possible examples was neither performed nor intended. We propose that our considerations apply regardless of whether the efficacy of combination partners is additive, less than additive or even more than additive. 


\section{General considerations, current practice, and regulatory recommendations}

\subsection{General considerations}

A meaningful combination treatment should fulfill several general criteria. Firstly, the co-administered drugs typically should not affect the pharmacokinetic profile of each other to avoid pharmacokinetic drug-drug-interactions. Exceptions are combinations explicitly designed that way such as the combination of a $\beta$-lactam antibiotic and a lactamase inhibitor to yield greater exposure to the $\beta$-lactam, for instance amoxicillin + clavulanic acid. Second, the drugs being combined should not exhibit additive toxicology as also highlighted by regulatory guidelines [7]. Third, the combination partners should exhibit compatible pharmacokinetic profiles, particularly if administration in a single pill is intended as a fixed-dose combination (FDC).

\subsection{Paralell group vs. add-on study designs}

Most evaluations of potential drug combinations are based on one of two types of study design. One type uses parallel groups of one or more monotherapies and combination treatment and has, for instance been applied to compare the effects of an $\alpha_{1}$-adrenoceptor antagonist, a $5 \alpha$-reductase inhibitor, and their combination in the treatment of male LUTS [8-12]. An advantage of this approach is that it is not biased by considerations which drug to administer first and which to add. However, a disadvantage is that starting all regimens in parallel does not allow determining which individuals (patients) need combination therapy, i.e., also includes subjects who already show a sufficient response to one of the monotherapies. Thus, this approach is suitable to explore whether addition of a novel drug provides benefit on top of standard of care. It is also suitable when effects on long-term, particularly major outcomes such as mortality are investigated, where waiting for non-responder status would be too late, unethical and/or unpractical for time-line reasons. For instance, a parallel group design this has been used to determine whether the sodium-glucose transporter 2 inhibitors dapagliflozin [13] and empagliflozin [14] reduce mortality and other cardiovascular endpoints in patients with heart failure with reduced rejection fraction when added on top of standard of care.

A second approach is based on selecting insufficient responders to a monotherapy and then giving a second drug as add-on treatment. The main benefit of this approach is that it focuses on patients with an insufficient response to monotherapy, i.e., those who may need combination treatment. In its simplest way, this can be done by open-label addition of a second drug. This has been applied for instance in a study exploring effects of adding the $\beta_{3}$-adrenoceptor agonist mirabegron to the treatment of male LUTS with the $5 \alpha$-reductase inhibitor dutasteride [15]. Its key limitation is that the interpretation of outcomes is limited by various biases including a possible placebo effect and/or observer bias. Thus, in this example studies comparing mirabegron alone with placebo have demonstrated that approximately $60 \%$ of the response were also seen in the placebo group [16]. Of note, the placebo effect itself exhibits a 'dose-response' curve: in placebo-controlled dose-escalation studies, given a second placebo to those exhibiting limited treatment effect to a single dose increased the perceived effect [17]. Because of its limitations, this design can be informative in the context of a pilot study but should not be seen as more than generating a signal that a combination may be worth another look. Another possible application of this approach is open-label, post-marketing authorization studies in which non-responders to an established drug are given a recently approved drug, for instance mirabegron on top of the muscarinic receptor antagonist solifenacin [18]. This approach can be useful if the benefit of the combination has already been established and investigators wish to determine how this manifests in real-life practice.

To establish the efficacy of a combination as compared to monotherapy, it is more informative to randomize the insufficient responders to either continue with monotherapy or to receive the second drug, as reported for instance in a study where consecutive men with LUTS receiving the $\alpha_{1}$-adrenoceptor antagonist tamsulosin and not exhibiting a sufficient improvement were randomized to additionally receive the muscarinic receptor 
antagonist tolterodine or not [19]. While this approach reduces biases to some extent by having a parallel control group, it remains open to vulnerability by placebo effect and observer bias and, if anything is also largely limited to pilot studies. Therefore, the gold standard of the add-on design is to randomize insufficient responders to receive placebo or the second drug in a double-blind manner. This yields trials of similarly high quality as other placebo-controlled, double-blind, randomized studies. Therefore, this is frequently applied in studies for regulatory purposes. Several variations of this approach exist, and all of them have been used in the clinical development program for a combination of mirabegron and solifenacin. For instance, it is possible to compare the effect of an add-on drug to both continuation (to control for time effects) and to a dosage increase in the first drug [20]. Alternatively, multiple doses of the second drug can be added in nonresponders to an established treatment; this creates the add-on form of a dose-selection study. This may be more effective in identifying the right dose of combination partners than a parallel group design; for instance, one study has used 12 parallel arms including 6 with various doses of the combination to establish optimal doses of combinations of mirabegron and solifenacin [21].

Thus, each of these designs for the evaluation of combination treatments has distinct advantages and limitations. Parallel group studies are easy to design but imply that some adequate responders to one drug are unnecessarily exposed to a second drug. However, they are important in settings where long-term outcomes such as progression of male LUTS $[11,12]$ or effects in conditions with a high mortality such as heart failure are studied $[13,14]$. On the other hand, various types of add-on designs can be appropriate to specifically target non-responders to one drug. It is telling that a single clinical development program for a combination of mirabegron and solifenacin has concomitantly used many of the above approaches. This may reflect an uncertainty which study design is most appropriate and/or the realization that each of them may have specific advantages and disadvantages.

\subsection{Additional considerations for study design}

Determining an appropriate study design for combination treatment is further complicated by the fact that the benefit of a combination may not be equally applicable at all time points, particularly if time-to-onset for the desired therapeutic effects can be expected to differ between the combination partners. For instance, combination of the $5 \alpha$-reductase inhibitor finasteride with the phosphodiesterase 5 inhibitor tadalafil as compared to finasteride + placebo yielded a group difference of 1.7, 1.4 and 1.0 International Prostate Symptom Score (IPSS) points after 4, 12 and 26 weeks, respectively [22]. This decline in difference was not unexpected because improvements by $5 \alpha$-reductase inhibitors are known to require 3-6 months to become noticeable whereas those of phosphodiesterase 5 inhibitors typically manifest within days. On the other hand, studies in men with LUTS and a study duration of up to 1 year reported that combination treatment with an $\alpha_{1}$-adrenoceptor antagonist (alfuzosin, doxazosin, terazosin) and a $5 \alpha$-reductase inhibitor (finasteride) was not more efficacious than monotherapy with the $\alpha_{1}$-adrenoceptor antagonist [8-10], whereas studies with a duration of at least 2 years found combination treatment (doxazosin/finasteride or tamsulosin/dutasteride) to be more efficacious than either monotherapy at time points later than 1 year after initiation of treatment $[11,12]$.

Another consideration is that statistical superiority of combination treatment as compared to monotherapy does not always translate into clinically relevant improvement. For instance, it is proposed that the improvement of the AUA Symptom Score/IPSS must be at least 3 points in order to be noticeable by a man with LUTS [23]. Other definitions of a responder in this indication are a reduction of the IPSS by $\geq 50 \%$ [9] or by $\geq 25 \%$ and $\geq 3$ points [12]. However, studies with the combination of tamsulosin and solifenacin reported that the combination treatment using 6 or $9 \mathrm{mg}$ solifenacin was statistically superior to monotherapy with tamsulosin based on about 300 patients per study arm, but the effect size was moderate at best (IPSS reduction by tamsulosin, tamsulosin $+6 \mathrm{mg}$ solifenacin and tamsulosin $+9 \mathrm{mg}$ solifenacin $6.2,7.0$ and 6.5 points, respectively), i.e., combination 
treatment providing improvement by 0.3-0.8 IPSS points at the group level [24]. Similarly, meta-analyses of various studies comparing the effect of a combination of an $\alpha_{1}$-adrenoceptor antagonist and a phosphodiesterase 5 inhibitor in men with LUTS also reported statistical superiority over either monotherapy, but the effect size estimate was only about 1 IPSS point at the group level $[25,26]$. Thus, benefits at the group level were less than those needed to be considered a responder at the individual patient level, regardless which definition of a responder was used.

\subsection{Recommendations from regulatory authorities}

Interestingly, regulatory agencies such as US Food and Drug Administration or the European Medicine Agency provide only limited guidance on the evaluation of combination treatments. Apparently, this is motivated by the idea that testing a novel drug as addon to standard of care should follow the same general considerations as any other trial evaluating novel treatments. Examples of these are studies in which effects of the sodiumglucose transporter 2 inhibitors were compared to placebo in patients with heart failure receiving standard of care treatment $[13,14]$.

A different situation emerges in the development of an FDC. Regulatory authorities have issued guidelines for the non-clinical including toxicological evaluation of drug combinations $[7,27]$ and the clinical development of FDCs [28]. The latter requires to justify the pharmacological and medical rationale for the combination for the intended therapeutic indication. Part of the rationale may be that an FDC reduces the number of administered doses and thereby improves patient adherence, but that alone is considered as insufficient rationale. Rather it is expected that the applicant demonstrates that the FDC improves the benefit/risk ratio by increasing efficacy and/or improving safety relative to at least one of the monotherapies. However, the guideline does not inform further on the most appropriate trial designs other than stating that it can be based on placebo-controlled add-on designs, substitution of existing treatment consisting of the two concomitantly administered monotherapies with the FDC, or on initial combination treatment compared to a reference treatment, i.e., all options mentioned above. A guideline for the co-development of a combination of two or more new drugs, i.e., applicable when neither has been approved as monotherapy, states that a comparing the combination to placebo is sufficient if studies on the individual components and/or a strong rationale for the combination based on non-clinical studies are provided [29].

\section{Treatment benefit at the group vs. individual patient level}

\subsection{Individual benefit/risk considerations}

The mechanistic concept underlying combination treatment is that two or more molecular targets and their signaling cascades are involved in the pathophysiology of a given condition, and that concomitant targeting of more than one of these pathways provides greater clinical improvement than acting on either alone. For instance, male LUTS are considered to involve a static component due to the enlargement of the prostate and a dynamic component due to contraction of prostate and urethral smooth muscle as mediated by $\alpha_{1}$-adrenoceptors [30]. Thus, addressing both molecular targets concomitantly can be expected to have greater effects than either monotherapy. This theory has been supported in large, double-blind, randomized, long-term ( $\geq 4$ years) clinical trials for the combination of $\alpha_{1}$-adrenoceptor antagonist and $5 \alpha$-reductase inhibitors [11,12]; of note, this combination did not provide benefit over monotherapy with the $\alpha_{1}$-adrenoceptor antagonist when study duration was $\leq 1$ year [8-10].

Almost all studies comparing combination treatment to monotherapy have been based on assessing data at the group level as mean or median difference between treatments. However, almost no treatment works in every patient, implying that some patients receiving combination treatment almost by definition will not experience benefit as compared to at least one of the monotherapies. For instance, studies comparing an $\alpha_{1}$-adrenoceptor antagonist, a $5 \alpha$-reductase inhibitor and their combination reported that $39-67 \%$ of 
all participants were non-responders to at least one of the monotherapies $[9,12]$. Thus, various individual fates may occur behind an enhanced efficacy of a combination treatment at the group level as illustrated in Figure 1. There may be some subjects that are nonresponders to each monotherapy; these are unlikely to benefit from treatment with the combination of the monotherapies. It is also possible that a patient is a non-responder to one monotherapy but a responder to the other; if that person receives combination treatment, he/she will benefit from the combination but probably would benefit as much if only receiving the alternative monotherapy. For instance, IPSS improvements in men with LUTS receiving treatment with a $5 \alpha$-reductase inhibitor typically are limited to those with large prostates and even in this group take at least 3-6 months to develop. Accordingly some studies evaluating the combination of a $5 \alpha$-reductase inhibitor and an $\alpha_{1}$-adrenoceptor antagonist and follow-up for $\leq 12$ months found that the $5 \alpha$-reductase inhibitor was only marginally better than placebo, whereas the $\alpha_{1}$-adrenoceptor antagonist improved IPSS considerably; combination treatment also cause a major improvement, but yielded almost identical results as monotherapy with the $\alpha_{1}$-adrenoceptor antagonist $[8,10]$. Accordingly, men not sufficiently served by the $\alpha_{1}$-adrenoceptor antagonist had no benefit from combination treatment in this specific setting (they had benefit at the group level in studies of $\geq 4$ years of duration $[11,12]$ ). The only group that can expect benefit from combination treatment includes those patients who are (at least to some degree) responders to both monotherapies (see next section).

On the other hand, combination treatment exposes all patients to potential harm from adverse effects of both medications, including the subjects not experiencing individual benefit. For instance, five large studies in men with LUTS comparing an $\alpha_{1}$-adrenoceptor antagonist (alfuzosin, doxazosin, tamsulosin or terazosin), a $5 \alpha$-reductase inhibitor (dutasteride or finasteride) and the combination of both active treatments administered for 6 to $>52$ months, ejaculatory abnormalities were observed more frequently in the combination than in any other group, in four of these studies even more often than could be expected based on an additive effect [8-12]. Of note, neither of these studies found an efficacy benefit of the combination as compared to the $\alpha_{1}$-adrenoceptor antagonist within the first 12 months of observation, indicating that for this comparison at least for the first year of treatment combination did not provide benefit but caused at least some degree of harm to some patients. The resulting benefit-risk assessment may be acceptable if greater individual benefit can be achieved, but less so if the benefit at the group level is at least partly due to combination treatment addressing non-responders to monotherapy. Thus, the individual benefit/risk ratio may be negative for some patients even if the treatment provides benefit at the group level.

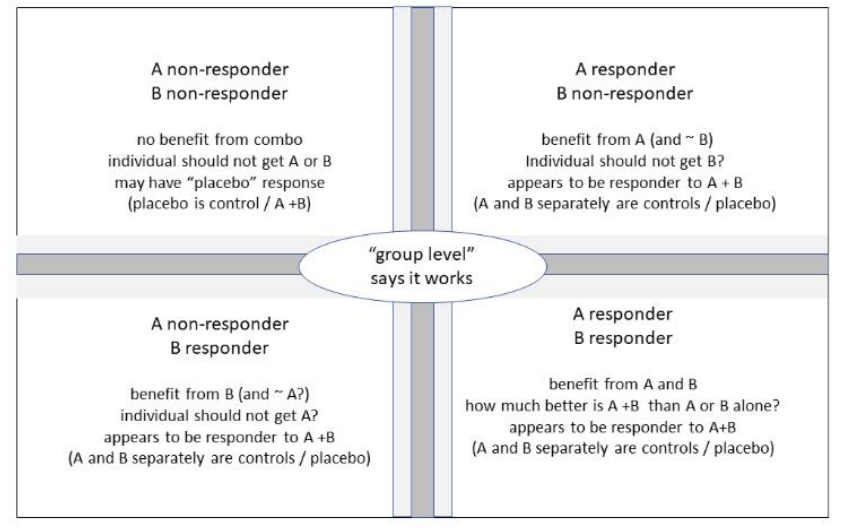

Figure 1. Schematic drawing of possible situations for individual patients if a drug works at the group level. Combination partners are indicated as drugs A and B. Note that this illustration is intentionally oversimplified to illustrate the concept that benefit at the population level does not necessarily imply benefit for each patient. 


\subsection{Expressing outcomes as responder rates and infering true beneficiaries of combination} treatment

Most studies evaluating combination treatments assess outcomes at the group level based on continuous variables that may or may not be normally distributed and correspondingly are expressed as means or medians [31]. However, each type of pharmacological treatment has responders and non-responders. While definitions of what constitutes a responder differ between indications or even within an indication such as male LUTS $[9,12,23]$, responder analysis may be informative in evaluating the effects of combination treatments and in discussing the probability of a beneficial treatment outcome with patients. In the following, we will provide arguments why expressing effects of combination treatment may be helpful.

The percentage of responder to two monotherapies A and B and their combination can be expressed as:

$$
\operatorname{Resp} \mathrm{T}=\operatorname{Resp} \mathrm{A}+\operatorname{Resp} \mathrm{B}-\operatorname{Resp} \mathrm{C},
$$

where Resp $\mathrm{T}$ is the percentage of responders in the overall group of subjects exposed to combination treatment, Resp A and Resp B the percentages of responders to treatments A and $B$, respectively, and Resp $C$ the percentage of subjects responding to both monotherapies (the subtraction of Resp $C$ is required because this group by definition includes parts of Resp A and Resp B). Of note, Resp A, Resp B and Resp T can directly be measured, whereas Resp C is a hypothetical value representing the group in which each member benefits from combination treatment because it only comprises members responsive to some extent to both monotherapies. If follows that all subjects not in the Resp C group do not benefit individually from combination treatment but are exposed to a risk for adverse events and thus potential harm. The percentage of subjects in this group P Harm can mathematically be defined as

$$
\text { P Harm }=100-\text { Resp C, }
$$

If Resp A, B and T are known, Resp C can be calculated by rearrangement of equation 1 as

$$
\operatorname{Resp} C=\operatorname{Resp} A+\operatorname{Resp} B-\operatorname{Resp} T,
$$

We can now calculate Resp T and P Harm based on various assumptions for Resp A, $B$ and $C$ using equations (1) and (2), and Resp C based on assumed or measured values of Resp A, B and T using equation (3). This has been done for several examples in Table 1. Readers can make their own assumptions and/or use measured values on responder rates in monotherapies and combination treatments based on data sets of their choosing using a spreadsheet provided as Online Supplement.

Table 1. Computation of Resp T and P Harm based on various assumptions for Resp A, B and C and of Resp I based on measured values of Resp A, B and T.

\begin{tabular}{ccccc}
\hline Resp A & Resp B & Resp C & Resp T & P Harm \\
\hline \multicolumn{5}{c}{ Assumed values of A, B and C } \\
50 & 50 & 0 & 100 & 100 \\
50 & 50 & 50 & 50 & 50 \\
70 & 70 & 50 & 90 & 50 \\
70 & 40 & 40 & 70 & 60 \\
70 & 40 & 30 & 80 & 70 \\
50 & 50 & 30 & 70 & 70 \\
50 & 50 & 10 & 90 & 90 \\
& Measured values of A, B and T & \\
43 & 33 & 34 & 42 & 66 \\
52 & 61 & 46 & 67 & 54 \\
\hline
\end{tabular}


For definitions see preceding paragraph. All data are shown in \% of patients exposed to combination treatment. Values in the last two rows are measured values for Resp A, B and T as reported in [9] and [12]. P Harm is always a calculated value.

The clinical challenge is that Resp C is the only group that truly benefits from combination treatment because each member is responsive to both combination partners, whereas all subjects outside of Resp C and thus by definition part of P Harm cannot expect individual benefit but nonetheless are exposed to adverse event risks associated receiving a drug that does not provide benefit to them. Although knowledge on Resp C is clinically very relevant, measuring it empirically is very challenging if not impossible. Equations (1) to (3) can be helpful to consider this issue conceptionally.

One extreme scenario is that the responder groups of the two monotherapies do not overlap at all and, therefore, no patient has individual benefit from the combination (see $1^{\text {st }}$ row of Table 1$)$. In this case, the apparent responder rate in the overall group $(\operatorname{Resp} \mathrm{T})$ would be $100 \%$ if responder rate to either monotherapy is $50 \%$. Nonetheless, the components of the combination treatment would have been ill chosen because no patient is likely to experience individual benefit beyond a placebo effect, but all patients receiving combination treatment are exposed to potential harm from receiving a second medication they do not need.

The other extreme scenario is that the two combination partners have almost identical responder groups ( $2^{\text {nd }}$ row of Table 1$)$, a scenario that is only realistic if the two drugs share the same molecular/cellular target. In that case, the components of the combination would also have been ill chosen (unless drug A had been underdosed) because the possible response from target $\mathrm{A}$ had already been fully exploited and given more of the same will only increase a risk for adverse events. Thus, P Harm mathematically would be the $50 \%$ not being a responder to either treatment in this example, but that would be an underestimate because also the responders would be exposed to a greater risk of harm without appreciable benefit. For instance, it had been found in a large study with a maximally effective dose of tamsulosin in men with LUTS that some patients received concomitant treatment with another $\alpha_{1}$-adrenoceptor antagonist (not a recommended treatment option); while the study did not report efficacy data in this small subgroup (17 out of 1784 men), it found that the odds ratio to experience an adverse event relative to the overall study population was 3.872 [95\% confidence interval 1.523; 9.847] [32]. Thus, optimal combination partners should be chosen to have distinct but overlapping responder groups.

The most realistic scenario is that the monotherapies at least partly address distinct responder groups that overlap to some extent. This overlapping responder group (Resp C) represents the true beneficiaries of combination treatment. Rows 3-7 of Table 1 represent various assumptions on the percentage of patients being responders to treatments $\mathrm{A}$ or $\mathrm{B}$ and to both monotherapies. These examples show that the measured responder rate in the combination arm always overestimates the percentage of subjects experiencing true benefit, the only exception being the unlikely scenario where both monotherapies have identical responder groups. In most scenarios, P Harm is larger than Resp C. How clinically relevant this is, depends of course on the severity and incidence of adverse effects of the two monotherapies, i.e., probably less relevant for a uroselective $\alpha_{1}$-adrenoceptor antagonist than for a cytotoxic drug used in cancer treatment.

Resp A, B and T can be measured in study designs where monotherapies and their combination are tested in parallel, and Resp C can be calculated from these values using equation (3). Two of the above studies testing the combination of an $\alpha_{1}$-adrenoceptor antagonist and a $5 \alpha$-reductase inhibitor in men with LUTS have reported responder rates for the monotherapies and their combination; that they had applied slightly different definitions what a responder is [9,12], is conceptually irrelevant to the present argument. One trial compared a 6-months treatment with alfuzosin, finasteride and their combination and found that $43 \%, 33 \%$ and $42 \%$ of them, respectively, were responders (not the primary endpoint of the study) [9]. Based on comparing Resp A and Resp T, it concluded that combination did not provide benefit. Another trial compared a 4-year treatment with tamsulosin, dutasteride and their combination and reported about $52 \%, 61 \%$ and $67 \%$ of 
patients to be responders, respectively [12], and concluded that combination treatment was superior to either monotherapy for IPSS improvement (neither IPSS improvement nor responder rates for such improvement were the primary endpoint of that study). Disappointingly, these data also demonstrate that $39-67 \%$ of patients were non-responders to one of the monotherapies and $33-57 \%$ were non-responders to combination treatment. Our calculations based on equation (3) show that the true percentage of patients benefitting from dual treatment is even lower, i.e., $34 \%$ and $46 \%$, respectively. Based on these data, we conclude that assessments at the group level are overly optimistic with regard to outcomes and thereby benefit/risk ratios at the individual patient level.

It may be argued that our above considerations are too pessimistic because we have treated responder status as a yes/no characteristics whereas it is a grey scale in clinical reality. This is obviously true but misses the point. While we have used responder status as a binary variable for simplicity of the argument, it of course can also be treated as a continuous variable; this requires a more complex set of mathematical assumptions and equations but would not in principle change the conclusion.

It can also be argued that making responder rates the primary outcome parameter can lead to a reduction of statistical power and, conversely, lead to a requirement of a greater sample size that makes a study more expensive and possibly requiring more time. Therefore, we explicitly do not recommend as a routine approach to use responder rate as the primary endpoint. However, it should be calculated and reported to enable.

Whether exposing patient to undue risk of harm because they have limited personal benefit due to receiving a drug that does not serve them personally, depends on specific aspects of seriousness of the condition to be treated on the one and severity and incidence of the adverse effects of a given treatment. Therefore, benefit/risk consideration will require medical judgment. However, we propose that understanding percentages of patients truly experiencing benefit from combination treatment (Resp C) and those not receiving benefit but being exposed to an additional drug (P Harm) will aid clinical decision making more than only looking at mean or median improvements at the group level.

\section{Conclusions}

We conclude that assessing benefits of combination treatment only at the group level over-estimates benefits to individual patients under almost all imaginable assumptions. Therefore, we propose that studies evaluating combination treatments should always report percentages of responders. The definition of a responder should of course be part of the study protocol and not set after data have been observed. The equations presented here may aid such efforts. Physicians should be skeptical if benefit at the group level is already small and of dubious clinical relevance because this implies that the percentage of patients truly benefitting from combination treatment is also small. Nonetheless, trying combination treatment in a given patient insufficiently response to treatment A may be an option under conditions where the existing and the added drug have a benign safety profile and possible benefit/improvement of the condition can be assessed in a short time.

While the above considerations aid the conceptual understanding of how to assess benefit/risk ratios of combination treatments, they fail to identify specific members of the hypothetical Resp C group. Doing so will require well validated and highly predictive biomarkers for the efficacy of either monotherapy. In a more general vein, investigators and physicians should focus on clinically meaningful effect sizes of monotherapies and combination treatments and not on statistical significance of minor effects in large cohorts.

Supplementary Materials: The following supporting information can be downloaded at: www.mdpi.com/xxx/s1, Online supplement 1: Excel sheet allowing calculations of Resp T and P Harm for any type of assumptions on Resp A, B, and I.

Author Contributions: Conceptualization, M.C.M. and D.S.; writing-original draft preparation, M.C.M.; writing - review and editing, D.S.; visualization, M.C.M. and D.S. All authors have read and agreed to the published version of the manuscript.

Funding: This research received no external funding. 
Institutional Review Board Statement: Not applicable.

Informed Consent Statement: Not applicable.

Data Availability Statement: Not applicable because this review does not report any previously undisclosed data.

Conflicts of Interest: M.C.M. is a consultant and/or lecturer to Apogepha, Astellas Pharma, Dr. Willmar Schwabe, GSK and Sanofi-Aventis. D.S. is a consultant, investigator and lecturer for Astellas Pharma and Urovant Sciences.

\section{References}

1. Weber, M.A.; Schiffrin, E.L.; White, W.B.; Mann, S.; Lindholm, L.H.; Kenerson, J.G.; Flack, J.M.; Carter, B.L.; Materson, B.J.; Ram, C.V., et al. Clinical practice guidelines for the management of hypertension in the community: a statement by the Amcerican Society of Hypertension and the International Society of Hypertension. J. Clin. Hypertens. 2014, 16, 14-26, doi:10.1111/jch.12237.

2. McDonagh, T.A.; Metra, M.; Adamo, M.; Gardner, R.S.; Baumbach, A.; Böhm, M.; Burri, H.; Butler, J.; Čelutkienė, J.; Chioncel, O., et al. 2021 ESC Guidelines for the diagnosis and treatment of acute and chronic heart failure. Eur. Heart J. 2021, in press, doi:10.1093/eurheartj/ehab368.

3. Cloutier, M.M.; Baptist, A.P.; Blake, K.V.; Brooks, E.G.; Bryant-Stephens, T.; DiMango, E.; Dixon, A.E.; Elward, K.S.; Hartert, T.; Krishnan, J.A., et al. 2020 Focused updates to the asthma management guidelines: a report from the National Asthma Education and Prevention Program Coordinating Committee Expert Panel working group. J. Allergy Clin. Immunol. 2020, 146, 1217-1270, doi:10.1016/j.jaci.2020.10.003.

4. $\quad$ Ettinger, D.S.; Wood, D.E.; Aggarwal, C.; Aisner, D.L.; Akerley, W.; Bauman, J.R.; Bharat, A.; Bruno, D.S.; Chang, J.Y.; Chirieac, L.R., et al. NCCN guidelines insights: non-small cell lung cancer, Version 1.2020. J. Natl. Compr. Canc. Netw. 2019, 17, 1464-1472, doi:10.6004/jnccn.2019.0059.

5. Lightner, D.J.; Gomelsky, A.; Souter, L.; Vasavada, S.P. Diagnosis and treatment of overactive bladder (non-neurogenic) in adults: AUA/SUFU Guideline Amendment 2019. J Urol 2019, 202, 558-563, doi:10.1097/ju.0000000000000309.

6. Gravas, S.; Cornu, J.N.; Gacci, M.; Gratzke, C.; Herrmann, T.R.W.; Mamoulakis, C.; Rieken, M.; Speakman, M.; Tikkinen, K.A. Management of non-neurogenic male LUTS. Availabe online: https://uroweb.org/guideline/treatment-of-nonneurogenic-male-luts/ (accessed on 22.12.2021).

7. European Medicines Agency. ICH guideline M3(R2) on non-clinical safety studies for the conduct of human clinical trials and marketing authoritzation for pharmaceuticals. Availabe online: https:/www.ema.europa.eu/en/documents/scientificguideline/ich-guideline-m3r2-non-clinical-safety-studies-conduct-human-clinical-trials-marketing-authorisation en.pdf (accessed on 25.12.2021).

8. Lepor, H.; Williford, W.O.; Barry, M.J.; Brawer, M.K.; Dixon, C.M.; Gormley, G.; Haakenson, C.; Machi, M.; Narayan, P.; Padley, R.J. The efficacy of terazosin, finasteride, or both in benign prostatic hyperplasia. N. Engl. J. Med. 1996, 335, 533-539.

9. Debruyne, F.M.; Jardin, A.; Colloi, D.; Resel, L.; Witjes, W.P.; Delauche-Cavallier, M.C.; McCarthy, C.; Geffriaud-Ricouard, C.; on behalf of the European, A.S.G. Sustained-release alfuzosin, finasteride and the combination of both in the treatment of benign prostatic hyperplasia. Eur. Urol. 1998, 34, 169-175, doi:10.1159/000019706.

10. Kirby, R.; Roehrborn, C.G.; Boyle, P.; Bartsch, G.; Jardin, A.; Cary, M.M.; Sweeney, M.; Grossman, E.B. Efficacy and tolerability of doxazosin and finasteride, alone or in combination, in treatment of symptomatic benign prostatic hyperplasia: the Prospective European Doxazosin and Combination Therapy (PREDICT) trial. Urology 2003, 61, 119-126, doi:10.1016/s0090-4295(02)02114-3.

11. McConnell, J.D.; Roehrborn, C.G.; Bautista, O.; Andriole, G.L.; Dixon, C.M.; Kusek, J.W.; Lepor, H.; McVary, K.T.; Nyberg, L.M.; Clarke, H.S., et al. The long-term effect of doxazosin, finasteride, and combination therapy on the clinical progression of benign prostatic hyperplasia. N. Engl. J. Med. 2003, 349, 2387-2398. 
Roehrborn, C.G.; Siami, P.; Barkin, J.; Damiao, R.; Major-Walker, K.; Nandy, I.; Morrill, B.B.; Gagnier, R.P.; Montorsi, F. The effects of combination therapy with dutasteride and tamsulosin on clinical outcomes in men with symptomatic benign prostatic hyperplasia: 4-year results from the CombAT study. Eur. Urol. 2010, 57, 123-131, doi:10.1016/j.eururo.2009.09.03.

13. McMurray, J.J.V.; Solomon, S.D.; Inzucchi, S.E.; Køber, L.; Kosiborod, M.N.; Martinez, F.A.; Ponikowski, P.; Sabatine, M.S.; Anand, I.S.; Bělohlávek, J., et al. Dapagliflozin in patients with heart failure and reduced ejection fraction. N. Engl. J. Med. 2019, 381, 1995-2008, doi:10.1056/NEJMoa1911303.

14. Packer, M.; Anker, S.D.; Butler, J.; Filippatos, G.; Pocock, S.J.; Carson, P.; Januzzi, J.; Verma, S.; Tsutsui, H.; Brueckmann, M., et al. Cardiovascular and renal outcomes with empagliflozin in heart failure. N. Engl. J. Med. 2020, 383, 1413-1424, doi:10.1056/NEJMoa2022190.

15. Maeda, T.; Kikuchi, E.; Hasegawa, M.; Ishioka, K.; Hagiwara, M.; Miyazaki, Y.; Shinojima, T.; Miyajima, A.; Oya, M. Solifenacin or mirabegron could improve persistent overactive bladder symptoms after dutasteride treatment in patients with benign prostatic hyperplasia. Urology 2015, 85, 1151-1155, doi:10.1016/j.urology.2015.01.028.

16. Chapple, C.R.; Cardozo, L.; Nitti, V.W.; Siddiqui, E.; Michel, M.C. Mirabegron in overactive bladder: a review of efficacy, safety, and tolerability. Neurourol. Urodyn. 2014, 33, 17-30, doi:10.1002/nau.22505.

17. Staskin, D.R.; Michel, M.C.; Sun, F.; Guan, Z.; Morrow, J.D. The effect of elective sham dose escalation on the palcebo response during an antimuscarinic trial for overactive bladder symptoms. J. Urol. 2012, 187, 1721-1726, doi:10.1016/j.juro.2011.12.052.

18. Yamaguchi, O.; Kikizaki, H.; Homma, Y.; Igawa, Y.; Takeda, M.; Nishizawa, O.; Gotoh, M.; Yoshida, M.; Yokoyama, O.; Seki, N., et al. Safety and efficacy of mirabegron as 'add-on' therapy in patients with overactive bladder treated with solifenacin: a post-marketing, open-label study in Japan (MILAI study). BJU Int. 2015, 1146, 612-622, doi:10.1111/bju.13068.

19. Athanasopoulos, A.; Gyftopoulos, K.; Giannitsas, K.; Fisfis, J.; Perimenis, P.; Barbalias, G. Combination treatment with an $\alpha$-blocker plus an anticholinergic for bladder outlet obstruction: a prospective, randomized, controlled study. J. Urol. 2003, 169, 2253-2256, doi:10.1097/01.ju.0000067541.73285.eb.

20. Drake, M.J.; Chapple, C.; Esen, A.A.; Athanasiou, S.; Cambronero, J.; Mitcheson, D.; Herschorn, S.; Saleem, T.; Huang, M.; Siddiqui, E., et al. Efficacy and safety of mirabegon add-on therapy to solifenacin in incontinent overactive bladder patients with an ilnadequate response to initial 4-week solifenacin monotherapy: a randomised double-blind multicentre phase 3B study (BESIDE). Eur. Urol. 2016, 70, 136-145, doi:10.1016/j.eururo.2016.02.030.

21. Abrams, P.; Kelleher, C.; Staskin, D.; Rechberger, T.; Kay, R.; Martina, R.; Newgreen, D.; Paireddy, A.; van Maanen, R.; Ridder, A. Combination treatment with mirabegron and solifenacin in patients with overactive bladder: efficacy and safety results from a randomised, double-blind, dose-ranging, phase 2 study (Symphony). Eur. Urol. 2015, 67, 577-588, doi:10.1016/j.eururo.2014.02.012.

22. Casabe, A.; Roehrborn, C.G.; Da Pozzo, L.F.; Zepeda, S.; henderson, R.J.; Sorsaburu, S.; Hennerges, C.; Wong, D.G.; Viktrup, L. Efficacy and safety of the coadministration of tadalafil once daily with finasteride for 6 months in men with lower urinary tract symptoms and prostatic enlargement secondary to benign prostatic hyperplasia. J. Urol. 2014, 191, 727-733, doi:10.1016/j.juro.2013.09.059.

23. Barry, M.J.; Williford, W.O.; Chang, Y.; Machi, M.; Jones, K.M.; Walker-Corkery, E.; Lepor, H. Benign prostatic hyperplasia specific healthy status measures in clinical research: how much change in the American Urological Association Symptom Index and the Benign Prostatic Hyerplasia Impact Index is perceptible to patients? J. Urol. 1995, 154, 1770-1774, doi:10.1016/S0022-5347(01)66780-6.

24. van Kerrebroeck, P.; Chapple, C.; Drogendijk, T.; Klaver, M.; Sokol, R.; Speakman, M.; Traudtner, K.; Drake, M.J. Combination therapy with solifenacin and tamsulosin oral controlled absorption system in a single table for lower urinary tract symptoms in men: efficacy and safety results from the randomised controlled NEPTUNE trial. European Urology 2013, 64, 1003-1012, doi:10.1016/j.eururo.2013.07.034. 
25. Sun, Y.; Peng, B.; Lei, G.-L.; Wei, Q.; Yang, L. Study of phosphodiesterase 5 inhibitors and a-adrenoceptor antagonists used alone or in combination for the treatment of lower urinary tract symptoms due to benign prostatic hyperplasia. Minerva Urol. Nefrol. 2020, 72, 13-21, doi:10.23736/S0393-2249.19.03408-8.

26. Kallidonis, P.; Adamou, C.; Kotsiris, D.; Ntasiotis, P.; Verze, P.; Athanasopoulos, A. Combination therapy with alphablocker and phosphodiesterase-5 inhibitor for improving lower urinary tract symptoms and erectile dysfunction in comparison with monotherapy: a systematic review and meta-analysis. Eur Urol Focus 2020, 6, 537-558, doi:10.1016/j.euf.2019.05.007.

27. U. S. Food and Drug Administration. Guidance for industry. Nonclinical safetry evaluation of drug or biologic combinations. Availabe online: https://www.fda.gov/media/119657/download (accessed on 25.12.2021).

28. European Medicines Agency. Guideline on clinical development of fixed combination medical procuts. Availabe online: https://www.ema.europa.eu/en/documents/scientific-guideline/guideline-clinical-development-fixed-combinationmedicinal-products-revision-2_en.pdf (accessed on 25.12.2021).

29. U.S. Food and Drug Administration. Guide for industry. Codevelopment of two or more new investigational drugs for use in combination. Availabe online: https://www.fda.gov/media/80100/download (accessed on 25.12.2021).

30. Madsen, F.A.; Bruskewitz, R.C. Benign prostatic hyperplasia: pathophysiology and pharmacological treatment. Curr. Opin. Nephrol. Hypertens. 1995, 4, 455-459.

31. Amiri, M.; Murgas, S.; Stang, A.; Michel, M.C. Do overactive bladder symptoms and their treatment-associated changes exhibit a normal distribution? Implications for analysis and reporting. Neurourol. Urodyn. 2020, 39, 754-761, doi:10.1002/nau.24275.

32. Michel, M.C.; Bressel, H.U.; Goepel, M.; Rübben, H. A 6-months large-scale study into the safety of tamsulosin. Br. J. Clin. Pharmacol. 2001, 51, 609-614, doi:10.1046/j.0306-5251.2001.01388.x. 